\title{
hTERT mRNA dendritic cell vaccination: complete response in a pancreatic cancer patient associated with response against several hTERT epitopes
}

\author{
Else M. Inderberg Suso - Svein Dueland • Anne-Marie Rasmussen • \\ Turid Vetrhus · Steinar Aamdal • Gunnar Kvalheim • \\ Gustav Gaudernack
}

Received: 19 November 2010/ Accepted: 8 February 2011/Published online: 2 March 2011

(C) The Author(s) 2011. This article is published with open access at Springerlink.com

\begin{abstract}
Immunotherapy targeting the hTERT subunit of telomerase has been shown to induce robust immune responses in cancer patients after vaccination with single hTERT peptides. Vaccination with dendritic cells (DCs) transfected with hTERT mRNA has the potential to induce strong immune responses to multiple hTERT epitopes and is therefore an attractive approach to more potent immunotherapy. Blood samples from such patients provide an opportunity for identification of new, in vivo processed T-cell epitopes that may be clinically relevant. A 62-yearold female patient underwent radical surgery for a pancreatic adenocarcinoma. After relapse, she obtained stable disease on gemcitabine treatment. Due to severe neutropenia, the chemotherapy was terminated. The patient has subsequently been treated with autologous DCs loaded with hTERT mRNA for 3 years. Immunomonitoring was performed at regular intervals following start of vaccination and clinical outcome measured by CT and PET/CT evaluation. The patient developed an immune response
\end{abstract}

E. M. I. Suso · A.-M. Rasmussen · G. Gaudernack ( $\bowtie)$ Section for Immunology, Oslo University Hospital and University of Oslo, Radiumhospitalet, Montebello, 0310 Oslo, Norway

e-mail: gustav.gaudernack@ rr-research.no

S. Dueland · S. Aamdal

Department of Clinical Cancer Research, Oslo University

Hospital, Radiumhospitalet, Oslo, Norway

T. Vetrhus

Department of Radiology, Oslo University Hospital,

Radiumhospitalet, Oslo, Norway

G. Kvalheim

Department of Cellular Therapy, Oslo University Hospital, Radiumhospitalet, Oslo, Norway against several hTERT-derived Th and CTL epitopes. She presently shows no evidence of active disease based on PET/CT scans. No serious adverse events were experienced and the patient continues to receive regular booster injections. We here provide evidence for the induction of hTERT-specific immune responses following vaccination of a pancreas cancer patient with DCs loaded with hTERT mRNA. These responses are associated with complete remission. A thorough analysis of this patient immune response has provided a unique opportunity to identify novel epitopes, associated with clinical effects. These will be included in future hTERT vaccines.

Keywords Telomerase (hTERT) - Epitope identification · Dendritic cell vaccination $\cdot$ Pancreas cancer

\section{Introduction}

Pancreatic adenocarcinoma is ranked fourth as a cause of cancer death world wide and is regarded as an essentially incurable disease. Patients with non-resectable, localized disease treated with chemotherapy have a median survival of 8-10 months [1] At best, chemotherapy prolongs survival for a few months. Thus, new treatment modalities are urgently needed. Immunotherapy may represent alternative treatment modalities and are presently under study in many cancer centres.

The rapid tumour progression followed by deterioration of the immune system is characteristic for patients with pancreas cancer. This argues for selection of patients for immunotherapy, amongst those where an initial stabilization of the disease by chemotherapy has been obtained. In this way, the immune system may have a chance to develop and consolidate the initial therapeutic response and possibly induce long-term survival benefit. 
Several cancer vaccines targeting well-defined antigens in pancreatic cancer have been tested in the clinic [2]. Telomerase (hTERT) is an attractive antigen target applicable also against pancreatic cancer [3]. Moreover, telomerase is expressed in cancer stem cells and targeting this antigen could therefore be an important tool to eliminate these cells which are not easily killed by conventional therapy [4].

The most widely used approach for the identification of novel tumour antigen targets is reverse immunology where epitopes are predicted in silico and then validated experimentally [5]. The concept is rational, but remains a cumbersome and complex process where approximately $50 \%$ of candidate peptides are lost at every step after selection of a target sequence. This approach may also lead to the identification of target peptides that may not be the most relevant in a clinical setting. Less than $1 \%$ of the peptides encoded by a target sequence are estimated to be antigenic, immunogenic, processed and presented on the cell surface and able to induce specific tumour lysis. We have here been able to use a direct approach immunising the patient with full-length hTERT mRNA and identifying the hTERT peptides capable of inducing patient T-cell responses post-vaccination.

Vaccination with mRNA-transfected DCs also offers the advantage of generating T-cell responses against multiple epitopes, involving both the CD4+ Th and CD8+ T-cell subsets. In the human system, DCs transfected with mRNA have previously been shown to induce CTL responses to hTERT in vitro [6]. Employed as treatment against metastatic prostate cancer, Su et al. also demonstrated successful generation of hTERT-specific T-cell responses measured as interferon- $\gamma($ IFN $-\gamma)$ secreting CD8 $+\mathrm{T}$ cells and CTLmediated killing of hTERT-positive targets [7]. Four patients experienced partial clinical responses. However, no hTERT epitopes were characterized in these studies.

Here, we have characterized the hTERT epitopes recognized by the patient $T$ cells following vaccination with hTERT-transfected DCs. Vaccination resulted in a diverse immune response involving both CD4+ Th cells and CD8+ $\mathrm{T}$ cells. This hTERT-specific T-cell response may be responsible for the effects such as the tumour regression and long-term survival observed. This opens up new avenues in novel peptide discovery for the design of efficient peptide vaccines.

\section{Methods}

Patient

A 62-year-old woman received surgery for a ductal adenocarcinoma of the pancreas. About 10 months later, she developed multiple metastatic lymph node lesions in the abdomen. She was treated with standard gemcitabine chemotherapy for 5 months and obtained stable disease on this treatment. The chemotherapy was cancelled due to severe neutropenia despite a $50 \%$ dose reduction. She was then vaccinated with DCs loaded with hTERT mRNA on a compassionate use basis following a standard clinical protocol used for patients with malignant melanoma. This protocol had been approved by the Norwegian Medicinal Authorities (SLV) and the regional ethical committee (REK). The patient had given informed consent and the treatment was performed according to the World Medical Association Declaration of Helsinki.

\section{Production of mRNA-transfected DCs}

DCs were generated as described earlier [8]. Briefly, monocytes obtained from leukapheresis product were cultured for 5 days with granulocyte-macrophage colonystimulating factor (GM-CSF) and interleukin-4 (IL-4) and then cultured for 2 days with cytokines facilitating maturation (interleukin-1 $\beta$ (IL-1 $\beta$ ), interleukin-6 (IL-6), tumour necrosis factor $\alpha(\mathrm{TNF}-\alpha)$ and prostaglandin $\mathrm{E}_{2}$ (PGE2). The resulting mature DCs were transfected (tDCs) with hTERT mRNA by square wave electroporation. As a control, a fraction of the DCs was mock transfected (no mRNA). The mature DC phenotype was evaluated by flow cytometry and shown to have high levels of HLA class II, CD86 and CD83, but not CD14. The DC viability was $>85 \%$, as assessed by trypan blue staining.

Fast DCs were generated for the second and third vaccine batches [9-11]. In brief, monocytes were cultured for 2 days with GM-CSF and IL-4 and then matured for 1 day in the same way as for conventional DC before electroporation. The DCs were then left overnight in the incubator prior to cryopreservation.

\section{Vaccine}

The vaccine consisted of $5 \times 10^{6}$ autologous monocytederived dendritic cells electroporated with hTERT mRNA. The patient received 4 weekly intradermal injections the first month followed by monthly booster injections. The first vaccine batch consisted of $5 \times 10^{6}$ conventional DCs and the patient had 15 vaccines administered. Batches 2 and 3 consisted of 10 and 17 vaccines, respectively, each containing $5 \times 10^{6}$ hTERT mRNA loaded fast DCs.

\section{Clinical monitoring}

Adverse events were recorded and graded according to the NCI common toxicity criteria, as previously reported [8]. Only minor side effects were observed, with no treatmentrelated grade III-IV toxicity. Objective tumour response was 
assessed by clinical examination and CT scans prior to start of vaccination and every 3 months during the vaccination. The tumour response was classified according to the Response Evaluation Criteria in Solid Tumours (RECIST) [12].

\section{Immunomonitoring}

Peripheral blood mononuclear cells (PBMCs) were obtained prior to the four standard vaccinations, after 5 weeks, after 12 weeks and before each monthly booster vaccination thereafter. The PBMCs were isolated and frozen as previously described [8]. Thawed PBMCs were stimulated once in vitro with tDCs, cultured and then tested in triplicates in T-cell proliferation assays $\left({ }^{3} \mathrm{H}\right.$-Thymidine) as previously described [8]. Antigen-presenting cells (APCs) were irradiated tDCs and mock DC controls. PBMCs from various time points were stimulated with an overlapping hTERT 15-mer peptide library or a 30-mer hTERT peptide (within the hTERT amino acid sequence 563-735, GenBank accession number: AB085628) and then tested in proliferation assays as above using irradiated PBMCs as APCs as already described [3]. All peptides were purchased from ProImmune (Oxford, UK). Stimulatory index (SI) is defined as proliferation with peptide divided by proliferation without peptide. SI $\geq 2$ is considered a positive response.

\section{Flow cytometry}

Pentamer staining was performed on fresh or frozen patient PBMCs. Phycoerythrin (PE)-conjugated pentamers were manufactured by ProImmune. Pentamer with HIV peptide SLYNTVATL-A*0201 was used as a negative control. Cells were stained with pentamers for $10 \mathrm{~min}$ at room temperature (RT), washed in staining buffer consisting of phosphate-buffered saline (PBS) containing $0.1 \%$ human serum albumin (HSA) and $0.1 \%$ sodium azide before staining with anti-CD4-fluorescein isothiocyanate (FITC), anti-CD19-FITC, anti-CD8-peridinin chlorophyll protein complex (PerCP) and anti-CD3-Pacific Blue (PB) (all from eBioscience San Diego, USA). FITC was used as a dump channel to exclude all CD4+ and CD19+ cells from the analysis. For intracellular staining, 12-day peptide-stimulated $\mathrm{T}$ cells were stimulated overnight in the presence of Brefeldin A (BD Bioscience, NJ, USA) at $10 \mu \mathrm{g} / \mathrm{ml}$ and BD Golgistop (BD Biosciences) at a 1/1,000 dilution with an autologous Epstein Barr Virus-transformed B-lymphoblastoid cell lines (EBV-LCL) loaded with peptide at a T cell-totarget ratio of 5:1. Non-peptide-loaded target cells and $\mathrm{T}$ cells alone were used as negative controls. Cells were then stained for CD4-PE-Cy7, CD8-PB, interferon-gamma (IFN- $\gamma$ )FITC (eBioscience), interleukin-2 (IL-2)-allophycocyanin (APC) (eBioscience) and tumour necrosis factor-alpha
(TNF- $\alpha$ )-PE (BD Bioscience) using the BD Cytofix/Cytoperm kit according to the manufacturer's instructions (BD Bioscience). Finally, cells were resuspended in staining buffer containing $1 \%$ paraformaldehyde. All antibodies and reagents for intracellular cytokine staining were purchased from BD Bioscience except where noted. Fifty to one hundred thousand CD8 $+\mathrm{T}$ cells were acquired per sample for the detection of pentamer-positive populations using a BD LSR II flow cytometer and the data were analysed using FlowJo software (Treestar Inc., Ashland, OR, USA).

\section{IFN- $\gamma$ ELISPOT}

The IFN- $\gamma$ ELISPOT assays were performed essentially as previously described [13]. Responder T cells were seeded in triplicates at $0.1 \times 10^{6} \mathrm{~T}$ cells/well and stimulated with irradiated, autologous PBMCs at a E:T ratio of 2:1. Long peptides (30- and 15 mers) 660-689, 663-677 and 673-687 were added at $25 \mathrm{uM}$ and 10 mer peptide 674-683 $10 \mathrm{uM}$. Negative controls with $\mathrm{T}$ cells only and positive controls stimulated with SEC-3 were included. Spots were enumerated using an automated analyzer, CTL IMMUNOSPOT S5 VERSA-02-9030 (Cellular Technology Ltd, Shaker Heights, USA).

\section{Results}

Clinical development and response

Following radical surgery and relapse, the patient experienced disease stabilization on gemcitabine treatment, but after 5 months the chemotherapy treatment was suspended due to adverse effects, neutropenia grade $2\left(1.0 \times 10^{9} / 1\right)$, despite a dose reduction of $50 \%$. She was then offered DC vaccination as an alternative treatment as described in the timeline (Fig. 1). She experienced a marked decrease in lymph node metastases ( $>30 \%$, partial response) as shown on CT scans obtained after 32 months of vaccination (Fig. 2a-c). Furthermore, there was no uptake on repeated

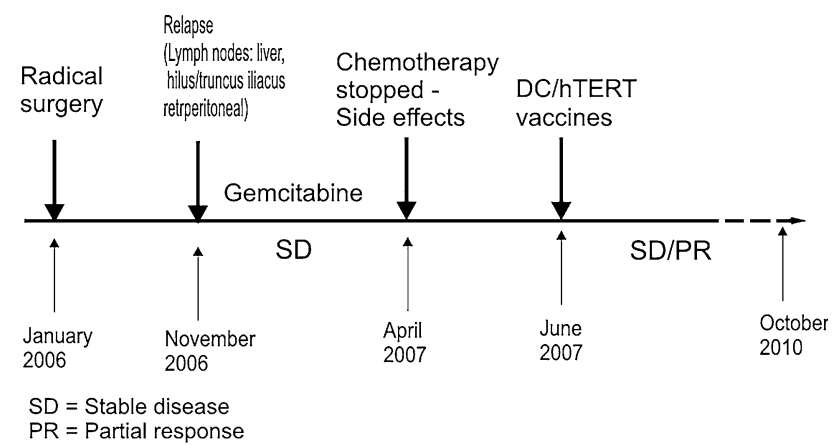

Fig. 1 Timeline for the clinical development of the pancreatic cancer patient treated with DC vaccine 
Fig. 2 CT pictures showing lymph node enlargement. a at the time of relapse before start of chemotherapy in November $2006(22.6 \mathrm{~mm}), \mathbf{b}$ at time of discontinued chemotherapy treatment in April 2007 $(26.3 \mathrm{~mm})$ and $\mathbf{c}$ after DC vaccination in February 2010 $(15.9 \mathrm{~mm})$
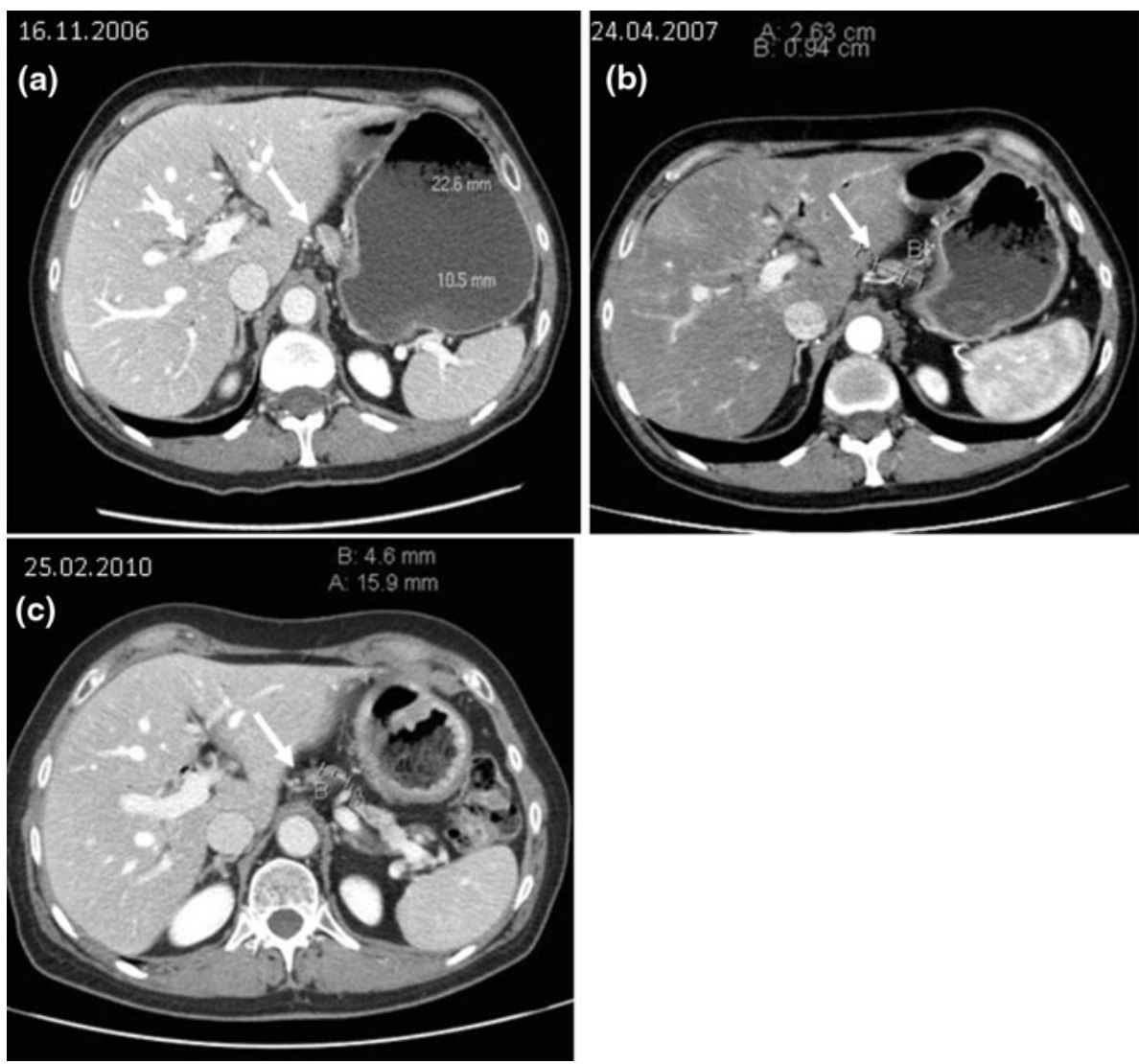

PET/CT scans indicating that the lesions have no increased metabolic activity compared to other lymph nodes. The patient has not experienced any serious treatment-related side effects during the 3-year vaccination period. Due to the demonstration of an immune response against the first DC vaccine batch and the presence of stable disease, new vaccine batches of fast DC were produced and immunomonitoring continued.

\section{hTERT-specific T-cell proliferation}

A proliferative T-cell response to the DC vaccine could be measured in vitro 3 months post-vaccination and was found stable from month 6 (data not shown). Having documented the presence of an immune response to hTERT-transfected DCs, we wanted to investigate which hTERT epitopes were responsible for inducing the immune response. This was tested by measuring T-cell proliferation against an hTERT peptide library (Fig. 3) and ex vivo pentamer staining of PBMCs. We detected proliferative T-cell responses $(S I \geq 2)$ against eight of the 15 -mer hTERT peptides from the overlapping peptide library and a 30-mer hTERT peptide at different time points throughout the vaccination period. The 30-mer peptide 660-689 was tested at later time points since it was synthesized after testing had started. Figure 3 shows a summary of the strongest responses detected against nine different peptides at various time points post-vaccination. The kinetics of the proliferative $\mathrm{T}$-cell responses against these nine peptides is presented in Table 1 with the first responses being detectable at 9 months after the start of vaccination. Importantly, all peptide-specific T-cell responses could be detected only post-vaccination, as shown graphically for four representative peptides in Fig. 4. Responses against most of these peptides became detectable in vitro only after several months of vaccination. This demonstrates the presence of hTERT-specific T-cell responses after vaccination and that some of these responses required repeated vaccination to develop to detectable levels.

\section{Pentamer analysis of hTERT peptide-specific T cells}

The ability of the vaccine to induce CD8+ T cells specific for HLA-A*0201 restricted hTERT peptides was assessed by ex vivo hTERT pentamer analysis of PBMCs harvested at different time points. A representative example of hTERT pentamer analysis from pre- and post-vaccination is shown in Fig. 5a. The top panel shows the presence of hTERT pentamer-positive populations of $0.12 \%$ of the CD8+ T cells for both the 10-mer peptide 674-683 pentamer and the 9-mer peptide 653-661 pentamer pre-vaccination. In the bottom panel at 24 months 
Fig. 3 Summary of hTERTspecific T-cell responses detected. T-cell proliferation against 24 overlapping hTERT 15-mer peptides were tested in samples from various time points throughout the vaccination schedule and responses against several hTERT peptides were detected. Proliferation was measured in response to peptide-loaded and non-loaded autologous PBMC and a stimulation index of $>2$ considered an immune response. Peptide sequences are shown on the x-axis. Black bars show peptides inducing T-cell responses and hatched bars peptides not inducing responses

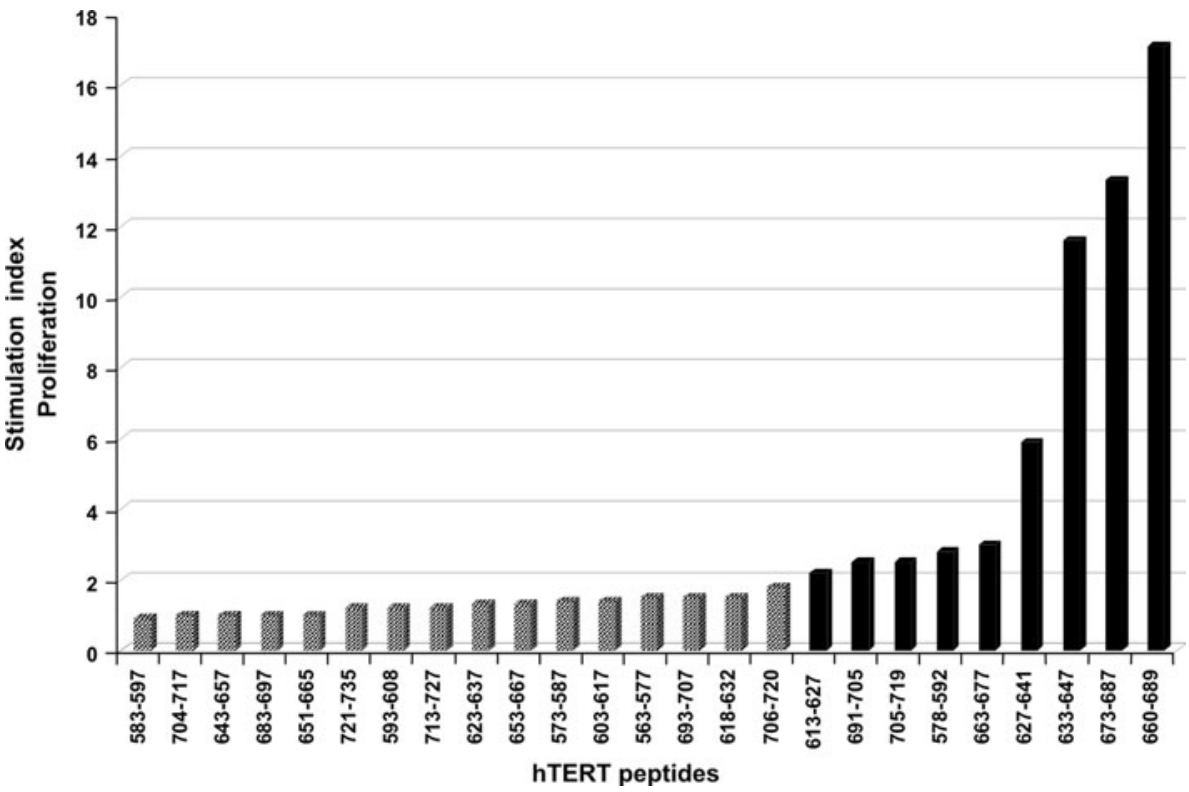

Table 1 Kinetics of peptide-specific T-cell proliferation at all time points tested

\begin{tabular}{|c|c|c|c|c|c|c|c|c|c|}
\hline \multirow[t]{2}{*}{ Time point } & \multicolumn{9}{|c|}{ hTERT peptides } \\
\hline & $613-627$ & $633-647$ & $663-677$ & $673-687$ & $578-592$ & $691-705$ & $705-719$ & $627-641$ & $660-689$ \\
\hline Baseline & $0.8^{\mathrm{a}}$ & 0.8 & 0.6 & 0.6 & 0.8 & 0.7 & 0.6 & 0.7 & $\mathrm{nt}$ \\
\hline 1 week & 0.5 & 0.8 & 0.6 & 0.6 & 0.5 & 0.6 & 0.6 & 0.6 & $\mathrm{nt}$ \\
\hline 6 weeks & 0.7 & 0.9 & 0.8 & 0.8 & 0.7 & 1 & 0.6 & 1 & $\mathrm{nt}$ \\
\hline 3 months & 1 & 1 & 0.8 & 0.8 & 1 & 0.7 & 0.8 & 0.7 & $\mathrm{nt}$ \\
\hline 4 months & 1.4 & 1 & 1 & 1 & 1.4 & 1.4 & 0.7 & 1.4 & $\mathrm{nt}$ \\
\hline 5 months & 0.9 & 0.7 & 1 & 1 & 0.9 & 1 & 0.4 & 1 & $\mathrm{nt}$ \\
\hline 6 months & 1.4 & 1.2 & 1.5 & 1.5 & 1.4 & 1.2 & 0.6 & 1.2 & $\mathrm{nt}$ \\
\hline 9 months & 2.1 & 0.8 & 0.7 & 1 & 2.7 & 1.3 & 1.1 & 1 & $\mathrm{nt}$ \\
\hline 17 months & nt & $\mathrm{nt}$ & 3.0 & 3.5 & nt & 2.5 & nt & 2.2 & $\mathrm{nt}$ \\
\hline 18 months & $\mathrm{nt}$ & $\mathrm{nt}$ & nt & nt & $\mathrm{nt}$ & nt & $\mathrm{nt}$ & nt & 3.1 \\
\hline 19 months & $\mathrm{nt}$ & $\mathrm{nt}$ & $\mathrm{nt}$ & $\mathrm{nt}$ & $\mathrm{nt}$ & $\mathrm{nt}$ & $\mathrm{nt}$ & $\mathrm{nt}$ & 2.1 \\
\hline 21 months & $\mathrm{nt}$ & $\mathrm{nt}$ & 1 & 1 & $\mathrm{nt}$ & 1.5 & $\mathrm{nt}$ & 0.5 & nt \\
\hline 22 months & $\mathrm{nt}$ & $\mathrm{nt}$ & 1.2 & 1 & $\mathrm{nt}$ & 1.2 & $\mathrm{nt}$ & 0.9 & $\mathrm{nt}$ \\
\hline 23 months & $\mathrm{nt}$ & $\mathrm{nt}$ & 1 & 0.9 & $\mathrm{nt}$ & 1 & $\mathrm{nt}$ & 1.2 & $\mathrm{nt}$ \\
\hline 24 months & 0.8 & 11.6 & 0.8 & 1.1 & 1.1 & 1.5 & 2.5 & 5.9 & 2.97 \\
\hline 30 months & nt & $\mathrm{nt}$ & 1.3 & 13.3 & $\mathrm{nt}$ & $\mathrm{nt}$ & $\mathrm{nt}$ & $\mathrm{nt}$ & 17.1 \\
\hline
\end{tabular}

$n t$ non-tested

${ }^{a}$ Proliferation is expressed as stimulation index (SI)

post-vaccination, $1.25 \%$ of the $\mathrm{CD} 8+\mathrm{T}$ cells are specific for the $674-683$ pentamer, whereas $0.13 \%$ bind the $653-661$ pentamer. These results together with the hTERT pentamer analysis at several other time points throughout the vaccination period (Fig. 5b), including a third hTERT pentamer (615-624), indicate that the CD8+ T cells specific for the 674-683 epitope expanded the most after vaccination. Pentamer-positive $\mathrm{T}$ cells were also detected pre-vaccination with increased frequencies for the 674-683 peptide at months 11, 17, 24, 30 and 32. The 674-683-specific T-cell population could be further increased to approximately $3 \%$ of CD8 + T cells after in vitro peptide stimulation (data not shown). The 674-683 epitope is part of the hTERT 30-mer peptide 660-689 which induced strong proliferative T-cell responses. The 653-661 epitope is embedded in the 15-mer sequence 651-665 shown not to induce detectable proliferative responses in Fig. 3. For peptide 653-661, the frequencies were much lower and only very slight increases 


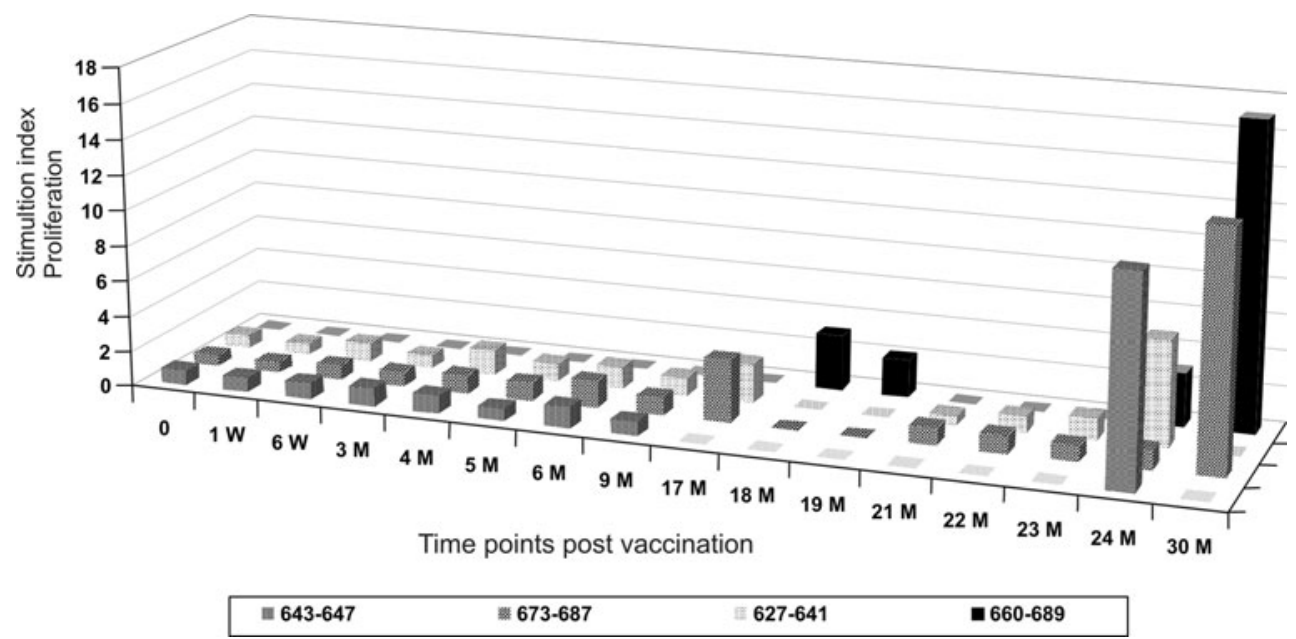

Fig. 4 Representative pre- and post-vaccination T-cell responses against hTERT peptides. T-cell responses against selected peptides throughout the vaccination schedule. Non-peptide-loaded PBMCs were used as negative controls. $W$ weeks and $M$ months post-start of vaccination. Flat grey squares represent time points where T-cell

were detected at months 17, 29, 30 and 32. Increased frequencies of $\mathrm{T}$ cells specific for the peptide 615-624 could in addition be detected at months 30 and 32 with $0.45 \%$ and $0.2 \%$ of the $\mathrm{CD} 8+\mathrm{T}$ cells being positive, respectively.

Cytokine secretion by hTERT peptide-specific T cells

After one round of in vitro stimulation of PBMCs with the 30-mer hTERT peptide 660-689 containing at least two T-helper epitopes (663-677 and 673-687) as well as the CTL epitope in the 674-683 pentamer, we could detect a population of multifunctional CD4+ T cells with a Th1type profile. These cells secreted IFN- $\gamma$, IL- 2 and TNF- $\alpha$ when cultured overnight with autologous Epstein Barr Virus (EBV)-transformed B cells loaded with the same peptide (Fig. 6a). There was no increase in cytokine production in the CD8+ T-cell population after stimulation with the peptide-loaded target cells compared to those stimulated with non-peptide-loaded targets (data not shown). High background levels of IL-2 observed may be due to the 12-day culture and to some stimulation given by the transformed B cell line which could express hTERT. hTERT peptide-specific IFN- $\gamma$ secretion was also detected by ELISPOT upon restimulation with 660-689 and nested epitope 673-687 after 12-day cultures with hTERT 30-mer peptide (shown at 30 months in Fig. 6b). Here, the CD4+ and $\mathrm{CD} 8+\mathrm{T}$-cell responses were not separated, but the CTL epitope did not induce IFN- $\gamma$ secretion much above the background level. Taken together, these data confirm that T-cell populations specific for multiple hTERT peptides have been induced after vaccination of this patient. responses against the peptide were not tested. Black bars represent responses against peptide 660-689, light hatched bars against peptide 627-641, more intense hatched bars against peptide 673-687 and grey hatched bars against peptide 643-647

\section{Discussion}

In this paper, we describe a pancreas cancer patient with an extraordinary disease course following treatment with chemotherapy and vaccination with autologous DC transfected with hTERT mRNA. In addition, we demonstrate how vaccination with full-length mRNA can be used for the discovery of novel, clinically validated T-cell epitopes.

The patient underwent radical surgery in January 2006. Due to post-operative complications, she did not receive adjuvant chemotherapy. The patient relapsed in November 2006 with enlarged lymph nodes localized in liver hilus, truncus iliacus and retroperitoneum as assessed by CT. The original diagnosis was confirmed by re-examination of the surgical specimen by an independent pathologist. The patient obtained stable disease on gemcitabine chemotherapy that was discontinued due to side effects. In this clinical setting, from June 2007, the patient has been vaccinated with autologous DC loaded with hTERT mRNA on a compassionate use basis in order to consolidate the beneficial effect of the chemotherapy.

Rather than developing progressive disease after ended chemotherapy, the patient experienced a partial response against the tumour. Two consecutive PET scans in March and September 2009 revealed no metabolically active lesions. These intriguing findings indicate that the immunotherapeutic strategy used has induced a clinically relevant immune response in this patient. It was therefore important to document and study in depth the immune response against hTERT. Furthermore, as there is a complete lack of information regarding the detailed immune 
Fig. 5 Pentamer analysis of hTERT-specific HLA-A*0201 restricted $\mathrm{CD} 8+\mathrm{T}$ cells in peripheral blood. PBMC were stained directly after isolation without prior antigen stimulation. CD4+ and CD19+ cells were excluded from the analysis and plots show pentamer staining in CD8+ $\mathrm{CD} 3+$ cells. HIV-pentamer is used as a negative control for non-specific staining.

a Pentamer staining for two novel hTERT peptides 674-683 and 653-661 is shown. The top panel shows pentamer analysis pre-vaccination and the bottom panel at 24 months after start of vaccination. b Kinetics of the frequency of pentamer-positive cells detected in PBMCs at different post-vaccination time points $(\mathrm{M}=$ months). Dark dotted bars represent HIVpentamer-positive cells, black bars represent 674-683 pentamer-positive cells, hatched bars represent 653-661 pentamer-positive cells and light dotted bars 615-624 pentamer-positive cells, respectively. At least 50,000 $\mathrm{CD} 8+\mathrm{T}$ cells were acquired and analysed in each test
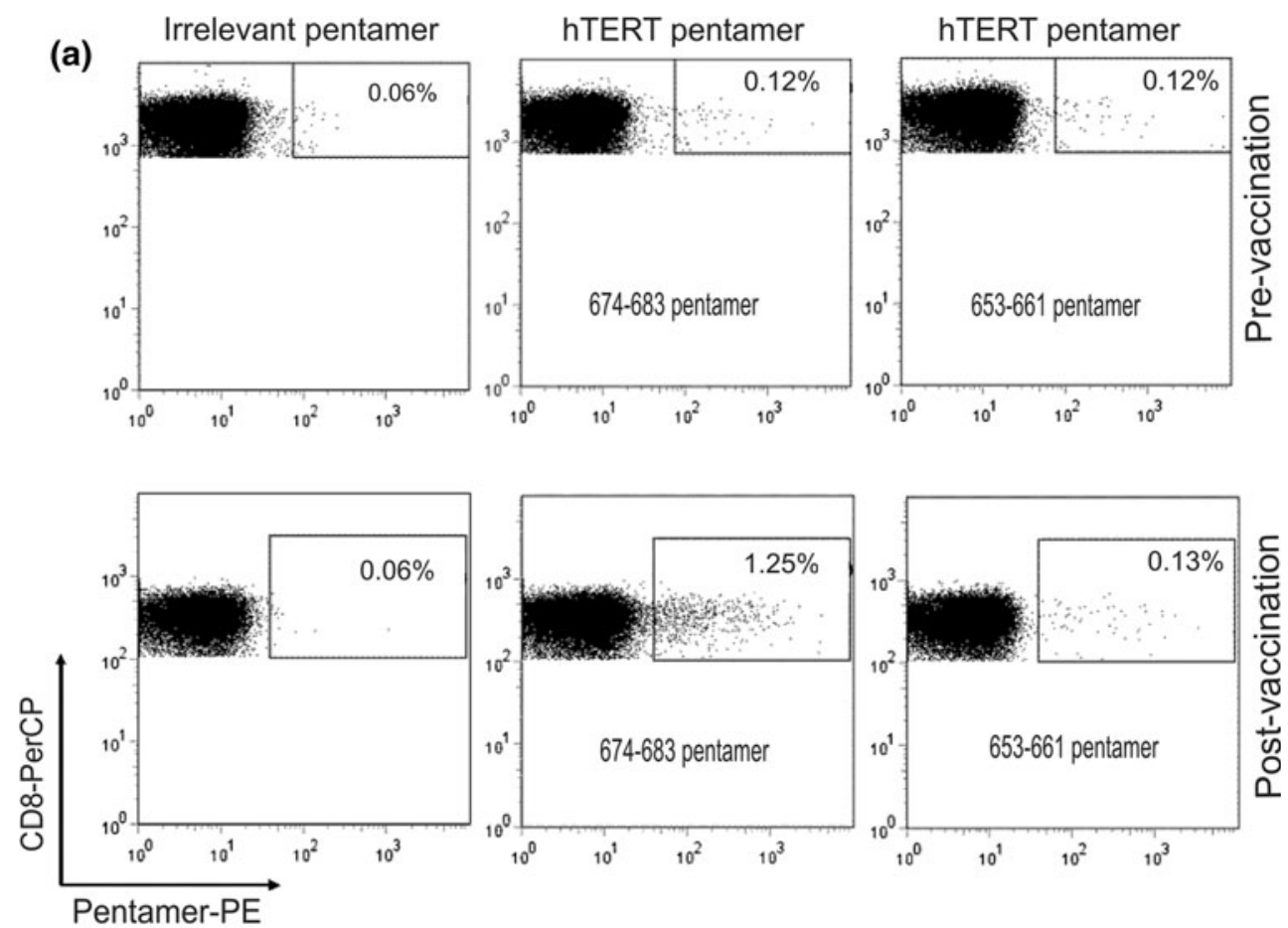

(b)

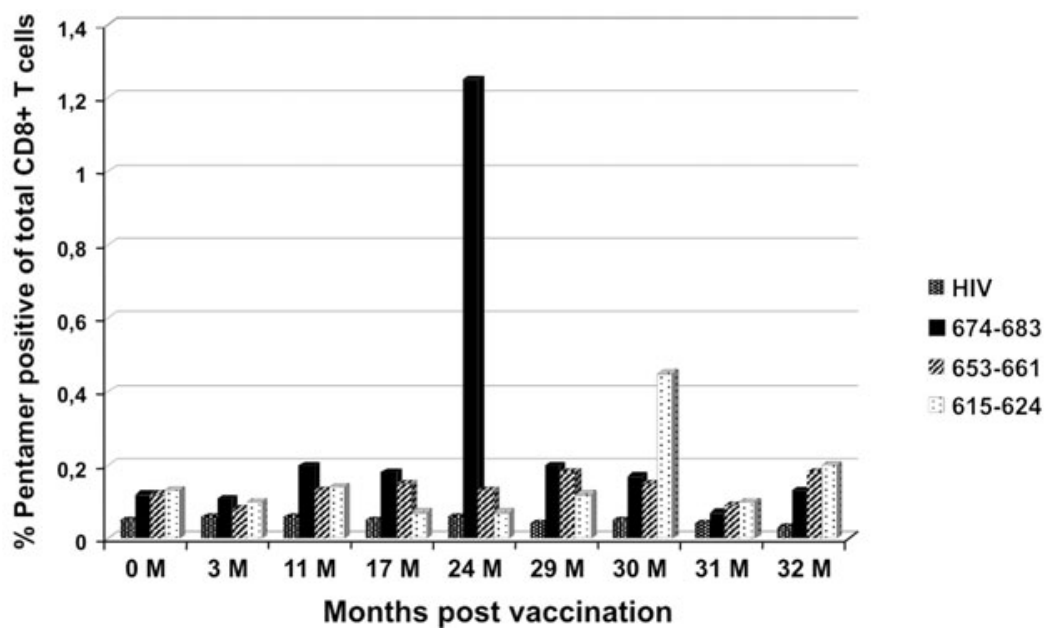

response against hTERT from studies using full-length hTERT mRNA for vaccination [7], it was important to identify hTERT epitopes potentially relevant in the antitumour response for the development of the next generation of hTERT vaccines.

We found high frequencies of CD8+ T-cell-binding pentamers with new CTL epitopes 674-683, but not 653-661 after vaccination. These two epitopes were HLAA*0201 restricted and have not been previously described. Interestingly, the 9-mer epitope 674-683 is embedded in a 15-mer peptide 673-687 nearly identical to the R672 Th epitope previously described by Schroers et al. [14]. Importantly, the same 15-mer peptide, 673-687, was also recognized by the $\mathrm{T}$ cells in proliferation assays, indicating that this region of the hTERT sequence may have elicited both a CD4+ and a CD8+ T-cell response in this patient. Moreover, seven other 15-mer peptides as well as one 30-mer peptide were recognized by $\mathrm{T}$ cells from this patient. Three of these peptides (663-677, 578-592 and 691-705) have not previously been reported. No immune responses against this set of peptides were observed when blood samples from six healthy donors were tested in the same assay (data not presented).

The amount of T-cell proliferation against these peptides was very variable. It is our general observation that immune responses against cancer vaccines in patients with concomitant tumours, as measured in peripheral blood, fluctuate considerably. This may reflect activated tumourspecific $\mathrm{T}$ cells leaving the blood stream to infiltrate the tumour. Stable immune responses are typically seen in 
Fig. 6 CD4+ T-cell cytokine secretion upon stimulation with a hTERT 30-mer peptide 660-689. PBMCs were cultured for 12 days in the presence of a hTERT peptide 660-689 containing several hTERT epitopes inducing T-cell proliferative responses. a The T-cell lines obtained from blood samples harvested 26 months post-vaccination start were restimulated overnight with autologous EBV-LCL loaded or not with peptide prior to intracellular cytokine staining. $\mathrm{T}$ cells were stained with CD4-PE-Cy7 or CD8-PB and IFN- $\gamma$-FITC, IL-2-APC and TNF- $\alpha$-PE. Plots show cells gated on CD4+ T cells. b Frequency of peptide-specific IFN- $\gamma$ secretion detected in ELISPOT assays from month 30 after start of vaccination. Autologous PBMCs were used as target cells. The E:T ratio was $2: 1$. Responses against peptides 663-667, 673-687, 660-689 and 674-683 were tested

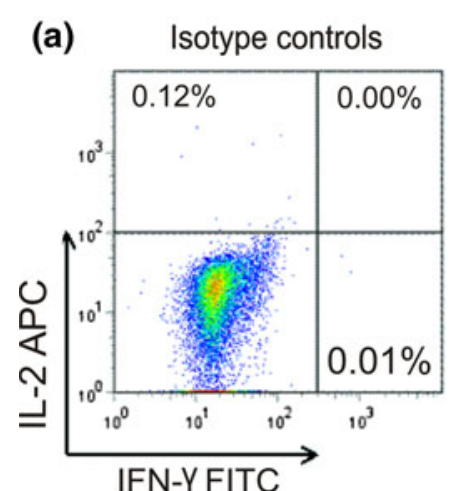

$\mathrm{TC}+\mathrm{EBV}-\mathrm{LCL}$ No peptide

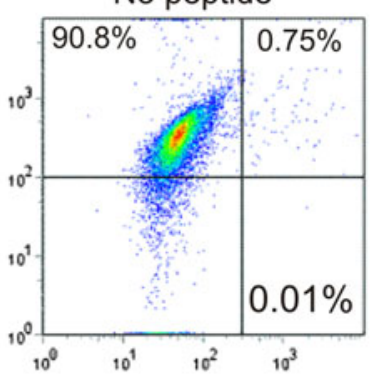

Tc + EBV-LCL hTERT peptide
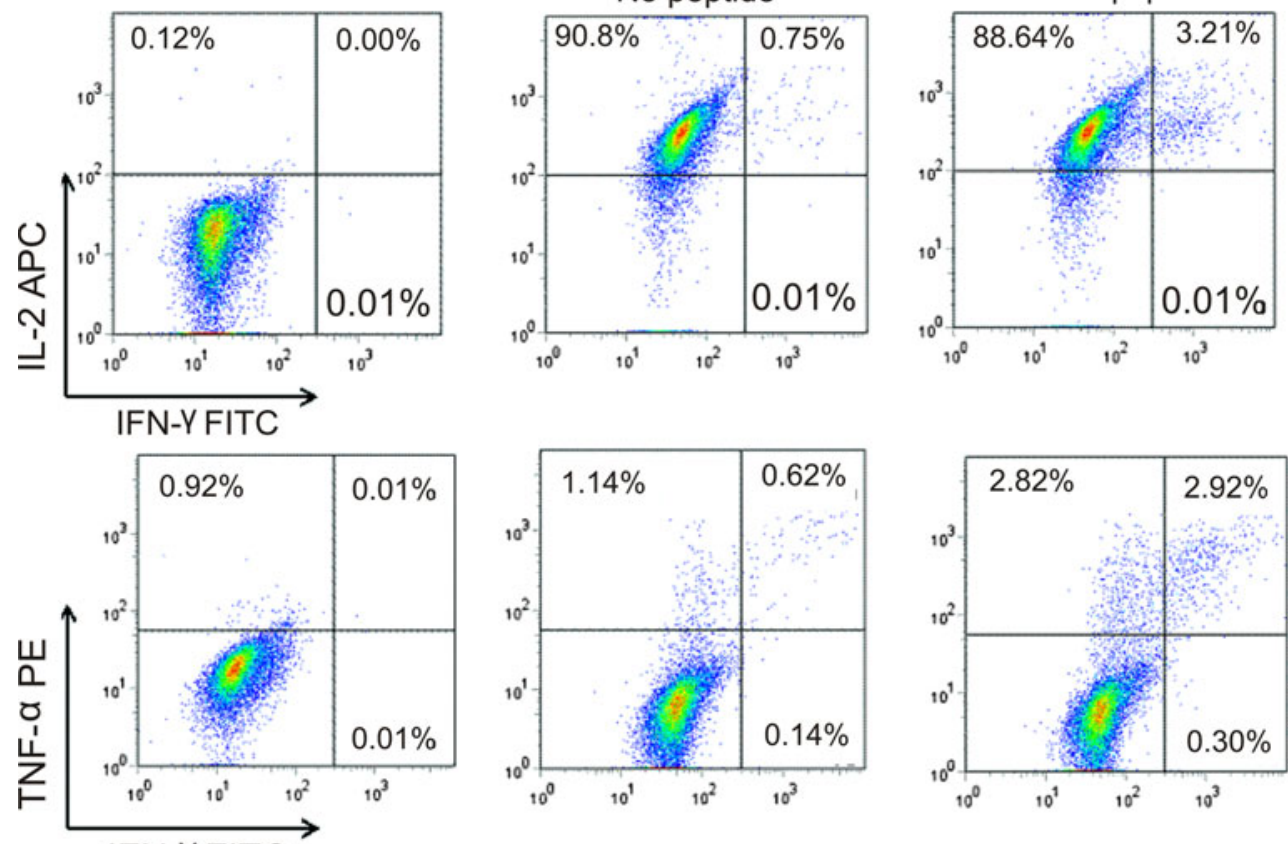

IFN-Y FITC

(b)

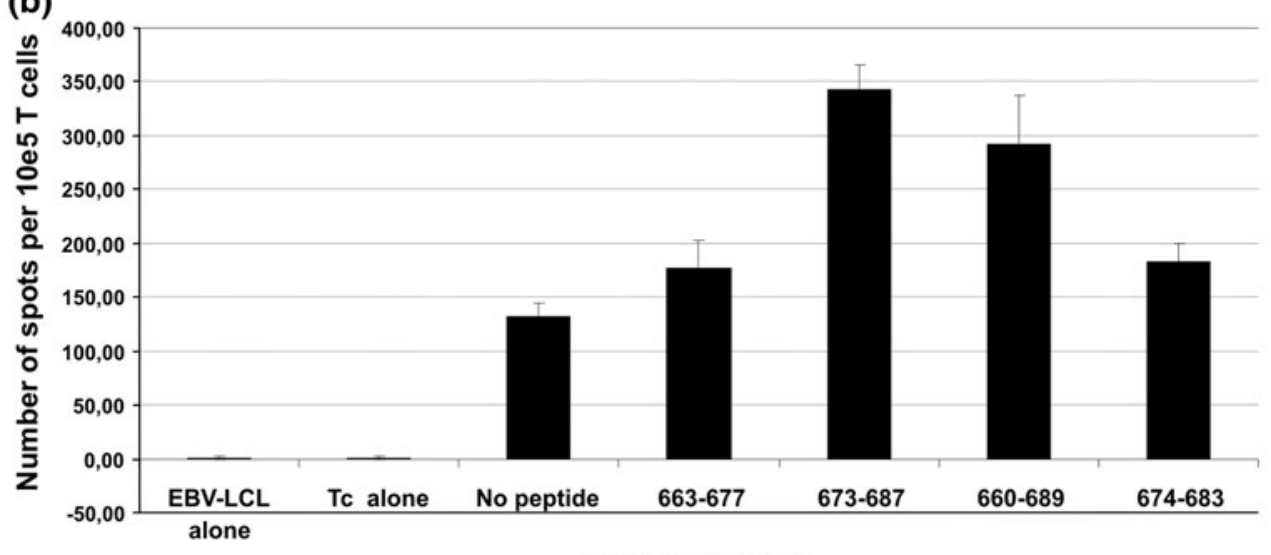

hTERT peptides

patients where complete responses have been obtained. Induction of new hTERT-specific $\mathrm{T}$ cells by repeated booster vaccinations will contribute to the dynamic immune response. Taken together, these results demonstrate that the vaccine has induced $\mathrm{T}$-cell responses against at least 10 different hTERT epitopes in this patient. The number of epitopes may be considerably greater as we have used only a limited number of peptides from an overlapping peptide library not covering the whole sequence as well as only a few pentamers limited to HLA-A*0201 presented peptides.

There is increasing emphasis on the pivotal role of CD4+ $\mathrm{T}$ cell in the anti-tumour response both in supporting CD8 $+\mathrm{T}$-cell responses and for their direct antitumour effects [15-18]. The observation of a CD4+ T-cell response against the 660-689 peptide, together with proliferative responses against several other peptides shown to be recognized by $\mathrm{Th}$ clones (Inderberg Suso et al., unpublished), in addition to CTL responses against several HLA-A*0201 restricted epitopes, supports the notion that a combination of T-helper (Th) and CTL epitopes should be used in future vaccination protocols. For this reason, the use of synthetic long peptides (SLP) in cancer vaccination is very promising. SLPs efficiently deliver antigen to DCs and are processed into both Th and CTL epitopes present in the peptide. The peptide presentation of SLPs which require internalization and intracellular processing has been shown to be prolonged compared to direct exogenous MHC class I molecule loading and presentation of short peptide not requiring processing [19]. The prolonged 
peptide presentation is particularly important if the T-cell epitope displays weak MHC class I binding.

In addition, vaccination with short peptides can lead to tolerance of the immunizing antigens rather than immunity unless ex vivo loading of DC is used [20,21]. Vaccinating with both CTL and Th epitopes has been shown to prevent this tolerance induction and the immunogenicity is further improved if these are included in one single hybrid peptide $[22,23]$. This could provide an explanation for the strong immune responses and some of the extraordinary clinical responses seen in cancer patients vaccinated with GV1001, a 16-mer hTERT peptide [3, 24]. This Th epitope also incorporates HLA-A2- and HLA-B7-binding peptide motifs, and the strength of CD4+ T-cell responses detected against the GV1001 peptide correlates well with patient survival. Vaccination with full-length mRNA encoding defined antigens will also induce immune responses to both T-helper and CTL epitopes as demonstrated by the results obtained here.

Vaccination in advanced stage cancer patients will often have limited therapeutic effect by itself due to a combination of increasing immune suppression and rapidly growing tumour. The therapeutic effect may be enhanced when used in conjunction with conventional chemotherapy or radiotherapy, which may affect both regulatory $\mathrm{T}$ cells and tumour growth. Prior to the start of vaccination, the patient described here was treated with gemcitabine which may contribute to the induction of a broader immune response as shown by Nowak et al. [25]. Furthermore, our observations indicate that there is no widespread tolerance against hTERT peptides and that the vaccination strategy using hTERT mRNA-transfected DCs is highly potent. Interestingly, despite the presence of a complex hTERTspecific immune response over a period of $>3$ years, no evidence of autoimmunity involving hTERT-positive normal cells, such as haematopoietic stem cells, was observed. This is in line with previous observations in patients with long-term immune responses following hTERT peptide vaccination [24] and animal experiments [26]. Our newly identified peptides add to the list of 26 hTERT peptides that have been shown to induce efficient immune responses against hTERT-positive tumour cells (reviewed in [27]).

We have also demonstrated that CD4+ T cells from this patient, capable of recognizing a 30-mer hTERT peptide (660-689) encompassing two of the 15-mer and one 9-mer peptide, produce three Th1-associated cytokines simultaneously. This kind of multifunctionality has previously been demonstrated to give better protection against infection [28]. Peptide-specific cytokine secretion from CD8+ T cells could not be detected by flow cytometry and may reflect low initial precursor frequency of this CD8+ T-cell population. This difference in CD4+ and CD8+ T-cell numbers could be further increased by a much stronger proliferation of the CD4+ T cells recognizing the Th epitopes from the 30-mer peptide than of CD8+ T cells in the 12-day culture. The IFN- $\gamma$ ELISPOT confirmed that cytokine secretion occurred mainly in response to Th epitopes.

In conclusion, these results illustrate the feasibility of vaccination with DCs loaded with mRNA encoding a defined antigen for the identification of immunogenic T-cell epitopes. The major advantage is that the identified epitopes have been processed and presented in vivo and therefore are potentially clinically relevant. In general, this type of vaccination provides an opportunity for direct and fast discovery of novel T-cell epitopes from any tumourspecific or tumour-associated antigen.

Acknowledgments We are grateful to Kirsti Hønnåshagen, Sissel Trachsel, for excellent technical assistance, Jon Amund Kyte, Lone Hegg and Kristin Byrkjeland Nauste for clinical follow-up of the patient and to Sébastien Wälchli for careful reading of the manuscript. This work was supported by the Norwegian Ministry of Health (Gene Therapy Grant).

Conflict of interest The authors declare that they have no conflict of interest.

Open Access This article is distributed under the terms of the Creative Commons Attribution Noncommercial License which permits any noncommercial use, distribution, and reproduction in any medium, provided the original author(s) and source are credited.

\section{References}

1. Philip PA, Mooney M, Jaffe D, Eckhardt G, Moore M, Meropol N, Emens L, O'Reilly E, Korc M, Ellis L, Benedetti J, Rothenberg M, Willett C, Tempero M, Lowy A, Abbruzzese J, Simeone D, Hingorani S, Berlin J, Tepper J (2009) Consensus report of the national cancer institute clinical trials planning meeting on pancreas cancer treatment. J Clin Oncol 27(33):5660-5669

2. Wong HH, Lemoine NR (2008) Biological approaches to therapy of pancreatic cancer. Pancreatology 8(4-5):431-461

3. Bernhardt SL, Gjertsen MK, Trachsel S, Moller M, Eriksen JA, Meo M, Buanes T, Gaudernack G (2006) Telomerase peptide vaccination of patients with non-resectable pancreatic cancer: a dose escalating phase I/II study. Br J Cancer 95(11):1474-1482

4. Bhagwandin VJ, Shay JW (2009) Pancreatic cancer stem cells: fact or fiction? Biochim Biophys Acta 1792(4):248-259

5. Viatte S, Alves PM, Romero P (2006) Reverse immunology approach for the identification of CD8 T-cell-defined antigens: advantages and hurdles. Immunol Cell Biol 84(3):318-330

6. Saeboe-Larssen S, Fossberg E, Gaudernack G (2002) mRNAbased electrotransfection of human dendritic cells and induction of cytotoxic $\mathrm{T}$ lymphocyte responses against the telomerase catalytic subunit (hTERT). J Immunol Methods 259 (1-2): 191-203

7. Su Z, Dannull J, Yang BK, Dahm P, Coleman D, Yancey D, Sichi S, Niedzwiecki D, Boczkowski D, Gilboa E, Vieweg J (2005) Telomerase mRNA-transfected dendritic cells stimulate antigenspecific CD8+ and CD4+ T cell responses in patients with metastatic prostate cancer. J Immunol 174(6):3798-3807

8. Kyte JA, Mu L, Aamdal S, Kvalheim G, Dueland S, Hauser M, Gullestad HP, Ryder T, Lislerud K, Hammerstad H, Gaudernack 
G (2006) Phase I/II trial of melanoma therapy with dendritic cells transfected with autologous tumor-mRNA. Cancer Gene Ther 13(10):905-918

9. Alldawi L, Takahashi M, Narita M, Ayres F, Tsukada N, Osman Y, Furukawa T, Aizawa Y (2005) Effect of prostaglandin E2, lipopolysaccharide, IFN-gamma and cytokines on the generation and function of fast-DC. Cytotherapy 7(2):195-202

10. Tanaka F, Yamaguchi H, Haraguchi N, Mashino K, Ohta M, Inoue H, Mori M (2006) Efficient induction of specific cytotoxic $\mathrm{T}$ lymphocytes to tumor rejection peptide using functional matured 2 day-cultured dendritic cells derived from human monocytes. Int J Oncol 29(5):1263-1268

11. Ho WY, Nguyen HN, Wolfl M, Kuball J, Greenberg PD (2006) In vitro methods for generating $\mathrm{CD} 8+\mathrm{T}$-cell clones for immunotherapy from the naive repertoire. $\mathrm{J}$ Immunol Methods 310(1-2):40-52

12. Therasse P, Arbuck SG, Eisenhauer EA, Wanders J, Kaplan RS, Rubinstein L, Verweij J, Van Glabbeke M, van Oosterom AT, Christian MC, Gwyther SG (2000) New guidelines to evaluate the response to treatment in solid tumors. European Organization for Research and Treatment of Cancer, National Cancer Institute of the United States, National Cancer Institute of Canada. J Natl Cancer Inst 92(3):205-216

13. Kyte JA, Kvalheim G, Aamdal S, Saeboe-Larssen S, Gaudernack G (2005) Preclinical full-scale evaluation of dendritic cells transfected with autologous tumor-mRNA for melanoma vaccination. Cancer Gene Ther 12(6):579-591

14. Schroers R, Huang XF, Hammer J, Zhang J, Chen SY (2002) Identification of HLA DR7-restricted epitopes from human telomerase reverse transcriptase recognized by CD4+ T-helper cells. Cancer Res 62(9):2600-2605

15. Hunder NN, Wallen H, Cao J, Hendricks DW, Reilly JZ, Rodmyre R, Jungbluth A, Gnjatic S, Thompson JA, Yee C (2008) Treatment of metastatic melanoma with autologous CD4+ T cells against NY-ESO-1. N Engl J Med 358(25):2698-2703

16. Welters MJ, Kenter GG, Piersma SJ, Vloon AP, Lowik MJ, Berends-van der Meer DM, Drijfhout JW, Valentijn AR, Wafelman AR, Oostendorp J, Fleuren GJ, Offringa R, Melief CJ, van der Burg SH (2008) Induction of tumor-specific CD4+ and CD8+ T-cell immunity in cervical cancer patients by a human papillomavirus type 16 E6 and E7 long peptides vaccine. Clin Cancer Res 14(1):178-187

17. Xie Y, Akpinarli A, Maris C, Hipkiss EL, Lane M, Kwon EK, Muranski P, Restifo NP, Antony PA (2010) Naive tumor-specific CD4(+) $\mathrm{T}$ cells differentiated in vivo eradicate established melanoma. J Exp Med 207(3):651-667

18. Nakanishi Y, Lu B, Gerard C, Iwasaki A (2009) CD8(+) T lymphocyte mobilization to virus-infected tissue requires CD4(+) T-cell help. Nature 462(7272):510-513
19. Faure F, Mantegazza A, Sadaka C, Sedlik C, Jotereau F, Amigorena $S$ (2009) Long-lasting cross-presentation of tumor antigen in human DC. Eur J Immunol 39(2):380-390

20. Toes RE, Offringa R, Blom RJ, Melief CJ, Kast WM (1996) Peptide vaccination can lead to enhanced tumor growth through specific T-cell tolerance induction. Proc Natl Acad Sci USA 93(15):7855-7860

21. Toes RE, van der Voort EI, Schoenberger SP, Drijfhout JW, van Bloois L, Storm G, Kast WM, Offringa R, Melief CJ (1998) Enhancement of tumor outgrowth through CTL tolerization after peptide vaccination is avoided by peptide presentation on dendritic cells. J Immunol 160(9):4449-4456

22. Bijker MS, van den Eeden SJ, Franken KL, Melief CJ, Offringa R, van der Burg SH (2007) CD8+ CTL priming by exact peptide epitopes in incomplete Freund's adjuvant induces a vanishing CTL response, whereas long peptides induce sustained CTL reactivity. J Immunol 179(8):5033-5040

23. Zeng G, Li Y, El-Gamil M, Sidney J, Sette A, Wang RF, Rosenberg SA, Robbins PF (2002) Generation of NY-ESO-1specific CD4+ and CD8+ T cells by a single peptide with dual MHC class I and class II specificities: a new strategy for vaccine design. Cancer Res 62(13):3630-3635

24. Brunsvig PF, Aamdal S, Gjertsen MK, Kvalheim G, MarkowskiGrimsrud CJ, Sve I, Dyrhaug M, Trachsel S, Moller M, Eriksen JA, Gaudernack G (2006) Telomerase peptide vaccination: a phase I/II study in patients with non-small cell lung cancer. Cancer Immunol Immunother 55(12):1553-1564

25. Nowak AK, Lake RA, Marzo AL, Scott B, Heath WR, Collins EJ, Frelinger JA, Robinson BW (2003) Induction of tumor cell apoptosis in vivo increases tumor antigen cross-presentation, cross-priming rather than cross-tolerizing host tumor-specific CD8 T cells. J Immunol 170(10):4905-4913

26. Danet-Desnoyers GA, Luongo JL, Bonnet DA, Domchek SM, Vonderheide RH (2005) Telomerase vaccination has no detectable effect on SCID-repopulating and colony-forming activities in the bone marrow of cancer patients. Exp Hematol 33(11):1275-1280

27. Liu JP, Chen W, Schwarer AP, Li H (2010) Telomerase in cancer immunotherapy. Biochim Biophys Acta 1805(1):35-42

28. Darrah PA, Patel DT, De Luca PM, Lindsay RW, Davey DF, Flynn BJ, Hoff ST, Andersen P, Reed SG, Morris SL, Roederer M, Seder RA (2007) Multifunctional TH1 cells define a correlate of vaccine-mediated protection against Leishmania major. Nat Med 13(7):843-850 